low national chlamydia prevalence (estimated prevalence in 2009-2010 was $1.7 \%$, 95\% CI $1.0 \%$ to $2.3 \%$ ) combined with small survey sample sizes results in unstable estimates limiting the ability to monitor trends in demographic subpopulations.

Discussion The current US chlamydia surveillance system does not provide valid and timely data to estimate disease burden and monitor trends in chlamydial infection. The use of multiple data sources is not sufficient to offset inherent biases in each surveillance method. Consequently, new approaches to monitoring chlamydia morbidity are needed. Sentinel surveillance may provide higher quality data and a more comprehensive understanding of chlamydia trends.

\section{P51 PATIENT-REPORTED EFFECTS OF VAGINAL LACTOSE AS A PREBIOTIC FOR BACTERIAL VAGINOSIS}

doi:10.1136/sextrans-2012-050601c.51

${ }^{1} \mathrm{~S}$ J Emery, ${ }^{2} \mathrm{M}$ J Tomlinson, ${ }^{3} \mathrm{I} \mathrm{D}$ Hansen. ${ }^{1}$ Singleton Hospital; ${ }^{2}$ Concept Healthcare Ltd, Reading, UK; ${ }^{3}$ LadyBalance ApS, Denmark

Background Bacterial vaginosis (BV) is a common, frequently recurrent condition typically treated with oral metronidazole. Using lactose as a vaginal prebiotic to support Lactobacilli growth and lactic acid production is a new approach to treating and preventing overgrowth of vaginal pathogens. A vaginal tablet containing lactose $1.2 \mathrm{mg}$ (LadyBalance ApS, Denmark) has been available in Denmark since 2004. For the treatment of BV, it is administered once daily for 1 -week; it can subsequently be used on alternate days to prevent recurrence and to maintain the vaginal environment.

Aims To evaluate the perceived effect of a lactose vaginal tablet (LVT) on vaginal health.

Methods Women who had used the LVT between 2005 and 2009 were invited to complete a web-based questionnaire.

Results In the 728 responders who had used the LVT, the most commonly reported reasons for use were vaginal discharge with/ without malodour $(73 \% / 18 \%)$, vaginal itching or irritation $(37 \%)$, and vaginal dryness (16\%). $90 \%$ of women with self-reported vaginal discharge with offensive odour reported improvement within 1 week. Improvement of symptoms within 1 week was also reported by $81 \%$ of women with vaginal discharge without odour, $83 \%$ with vaginal itching and irritation, and $76 \%$ with vaginal dryness. These effects were generally maintained in women who continued to use the product over longer periods (up to 1 year). Reported side effects were minor and included clear or powdery discharge.

Discussion An LVT offers the potential of a natural treatment for $B V$, vaginal itching, irritation and dryness and for protection against recurrent $\mathrm{BV}$ or vaginal candidiasis. Its lack of serious side effects and drug interactions could make it an attractive alternative to standard therapies. The clinical effectiveness and tolerability of the LVT require further investigation.

Conclusions The LVT was perceived as highly effective in treating a range of vaginal symptoms.

\section{P52 NEW TREATMENT GUIDELINE OF NEISSERIA GONORRHOEAE AND TEST OF CURE: HOW FEASIBLE IS IT?}

doi:10.1136/sextrans-2012-050601c.52

S Rodgers, M Murgartroyd, K Perez, M Kingston, ${ }^{*}$ V Lee. Manchester Royal Infirmary, Manchester, UK

Background Treatment failure with oral cephalosporins in gonorrhoea caused by multidrug resistance has been reported. The national guidelines were updated in 2011 due to reduced sensitivity to antimicrobials. Test of cure (TOC) is recommended in all cases. However data regarding timing of TOC is limited.

Aims To assess (1) the feasibility of implementing new treatment regimen and (2) the optimal time to perform TOC.

Method Retrospective case note review of patients diagnosed with gonorrhoea in a GUM clinic between 1 June 2011 and 30 November 2011 was carried out. Data including demographics, HIV status, sites of infection, treatment and TOC were analysed.

Results 271 (242M, 29F) patients were included. 202 were men who have sex with men (MSM) of which $24 \%$ were HIV positive. In MSM group, 87 were urine TMA positive $(95 \%$ had cultures performed), 115 positive pharyngeal TMA (97\% with cultures done), 115 positive rectal TMA (96\% had cultures done). 39 heterosexual males had positive urine TMA ( $94 \%$ with cultures done). 18 $(62 \%)$ females had positive cervical TMA, all with culture performed. 12 (41\%) pharyngeal, $5(17 \%)$ vulvo-vaginal and $3(10 \%)$ rectal were TMA positive; $58 \%, 60 \%$, and $33 \%$ of these had cultures performed respectively. First-line treatment was given in $96 \%$ of cases. Second line treatments were given mostly due to penicillin allergy. TOC was attended by $55 \%$ of patients. $67 \%$ of TOC were done within 20 days of treatment. Three of these were positive, 2 within 20 days and one at 57 days post treatment which was a re-infection.

Conclusions New treatment of gonorrhoea is generally accepted by patients. $4 \%$ received alternative treatments, which were valid and documented. The majority of patients (74\%) had culture performed prior to treatment. TOC uptake was low (55\%). However the majority were performed within 20 days of treatment. Follow-up strategies must remain a priority to increase rates of TOC. Further studies are required to determine optimum time for TOC.

\section{P53 PCR SCREENING TESTS FOR CHLAMYDIA TRACHOMATIS OR NEISSERIA GONORRHOEAE DO NOT REOUIRE A SECOND TEST TO CONFIRM: AN AUDIT OF PATIENTS ISSUED WITH EQUIVOCAL RESULTS}

doi:10.1136/sextrans-2012-050601c.53

M Hopkins,* G Smith, I J Hart, F Alloba. Royal Liverpool University Hospital, Liverpool, UK

Background UK testing algorithms for chlamydia or gonorrhoea should have a positive predicative value (PPV) $>90 \%$. Repeat of testing of screen positive samples might be required to achieve this. Patients issued with unconfirmed positive (equivocal) results are recalled to clinic to submit another sample.

Objectives To assess the clinical utility of supplementary PCRs following a positive PCR screening test result.

Methods Laboratory reports for Chlamydia trachomatis or Neisseria gonorrhoeae issued to GUM patients between April 2010 and April 2011 were reviewed retrospectively. Positive reports were routinely confirmed by supplementary PCRs and $N$ gonorrhoeae culture. Clinical records of patients with equivocal reports were retrieved to determine if infection was confirmed by a second sample on patient recall and the impact of this process on antibiotic management.

Results Over 15000 patients were tested during the study period. The prevalence of chlamydia and gonorrhoea was $972(5.75 \%)$ and $76(0.50 \%)$, respectively. A further 78 chlamydia and 2 gonorrhoea equivocal reports were issued. Only $56(72 \%)$ patients with an equivocal chlamydia report returned to clinic, and of these, only 41 $(73 \%)$ gave a second sample to retest. PPV of the PCR screening test was calculated at $98.0 \%$ and $97.5 \%$ for detection of chlamydia infection from urine and rectal swabs, respectively. Most patients accepted antibiotic treatment before infection status had been confirmed. Prevalence of gonorrhoea infection was low but PPV of the screening PCR remained high $(98.75 \%)$ 